\title{
Centrifugal microfluidics for cell analysis
}

\author{
Robert Burger, Daniel Kirby, Macdara Glynn, Charles Nwankire, \\ Mary O'Sullivan, Jonathan Siegrist, David Kinahan, Gerson Aguirre, \\ Gregor Kijanka, Robert A Gorkin III and Jens Ducrée
}

\begin{abstract}
Over the past two decades, centrifugal microfluidic systems have successfully demonstrated their capability for robust, high-performance liquid handling to enable modular, multipurpose lab-on-a-chip platforms for a wide range of lifescience applications. Beyond the handling of homogeneous liquids, the unique, rotationally controlled centrifugal actuation has proven to be specifically advantageous for performing cell and particle handling and assays. In this review we discuss technologies to implement two important steps for cell handling, namely separation and capturing/counting.
\end{abstract}

\section{Address}

Biomedical Diagnostics Institute, National Centre for Sensor Research, School of Physical Sciences, Dublin City University, Ireland

Corresponding author: Ducrée, Jens (jens.ducree@dcu.ie)

Current Opinion in Chemical Biology 2012, 16:409-414

This review comes from a themed issue on Analytical techniques

Edited by Shana $\mathbf{O}$ Kelley and Petra S Dittrich

For a complete overview see the Issue and the Editorial

Available online 9th July 2012

1367-5931/\$ - see front matter, (C) 2012 Elsevier Ltd. All rights reserved.

http://dx.doi.org/10.1016/j.cbpa.2012.06.002

\section{Introduction}

Selective manipulation, sorting and analysis/identification of biological cells are very important operations for clinical diagnostics as well as for research applications. Cell sorting technologies such as fluorescence and magnetically activated cell sorting (known as FACS and MACS, respectively) are well established and have been commercially available for decades. However, they remain complex, expensive and limited to use in rather sophisticated lab infrastructures. While there is a clear need for high throughput cytometers, for example, in centralized clinical laboratories, there is also a demand for compact and portable low-cost devices, particularly for applications in resource poor settings or in a general practitioner's office. This has led to considerable interest both from academia and industry to investigate microfluidic systems for cell sorting and analysis. The various reviews published over recent years underpin the importance and scope of microfluidic systems for cell handling. Andersson and van den Berg provided an outline of microfluidic systems for cellomics [1]. Microfluidic devices for cell handling and counting were surveyed by Erickson and $\mathrm{Li}$ [2], while $\mathrm{Huh}$ and colleagues reviewed miniaturized flow cytometers [3]. Recently, the application of microfluidics for single cell analysis has been investigated [4].

This review focuses on centrifugal microfluidics for cell based applications. Such microfluidics in rotating systems utilize the centrifugal, Coriolis and Euler force to transport and manipulate liquids through their interaction with microstructures. Figure 1 shows the forces experienced by a liquid plug on a rotating disc. We will not describe non-rotational centrifugal lab-on-a-chip technologies which, for instance, induce centrifugal force by conventional pumping around sharp bends $[5,6]$. Compared to more conventional microfluidic actuation principles such as pressure-driven flow, the centrifugal microfluidic 'labon-a-disc' platform offers a number of intrinsic advantages, especially for particle handling $\left[7,8^{\bullet \bullet}, 9^{\bullet \bullet}, 10\right]$ : centrifugation offers a selective, sedimentation based transport of cells, even under stopped-flow conditions; the underlying liquid handling scheme is very robust and simply actuated by a conventional spindle motor, thus eliminating the need for external pumps; the centrifugal actuation is widely independent of fluid properties such as viscosity, $\mathrm{pH}$ and conductivity, which is particularly beneficial for handling biological samples. Lastly, the modular nature of this approach cleanly separates the disc containing the microfluidic network from the driving and detection units. This allows the liquid handling chip to be disposable, which is of specific interest for testing potentially infectious samples.

In this review we present recent advances in cell handling and analysis systems on centrifugal platforms with an emphasis on:

1. Cell separation, concentration and purification.

2. Cell capture, assaying and counting.

\section{Cell separation, concentration and purification}

A common first step in the cell analysis process chain is cell separation, either to obtain a cell free liquid fraction (supernatant extraction), to retrieve cellular constituents in their entirety, or to separate specific target cells from a background population. 
Figure 1

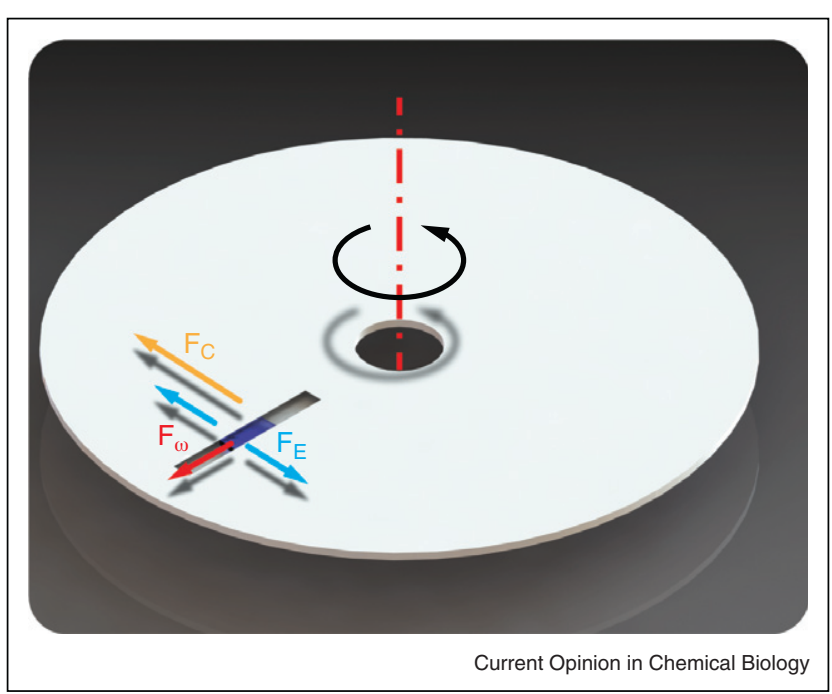

Forces acting on a liquid plug in a channel of a rotating disc. $F_{\mathrm{c}}=$ Coriolis force, $F_{\mathrm{E}}=$ Euler force and $F_{\omega}=$ centrifugal force.

The centrifugal platform readily lends itself to cell removal since cells typically have a higher density than the surrounding medium and they can hence be removed by sedimentation to yield a cell free supernatant. This is of particular interest for assays where cell free plasma needs to be extracted from whole blood. Zhang and coworkers developed a system to remove the cellular components from diluted blood using a curved channel [11]. Another blood separation system was presented by Häberle et al. [12]. In this work, blood has been preseparated while flowing through an azimuthally inclined throttling channel. Subsequently the cellular components have been collected in a first chamber while the cell free plasma was collected in an overflow chamber. Li et al. developed a blood separation structure comprising of two chambers connected by an out-of-plane valve to prevent the back-flow of cells into the plasma chamber [13]. The authors reported a plasma purity of up to $99.9 \%$. These technologies for complete cell removal are rather straightforward to implement on a centrifugal platform. More recently, research has been focused on the more challenging task of selectively separating target cells from a mixture of cells. On the one hand, this separation concentrates the target cells and thus reduces the sample volume which needs to be handled on the miniaturized chip in subsequent process steps. On the other hand, upstream separation simplifies and enhances subsequent detection of the target cells. Such a concentration step is particularly important in cases where the target cells are present in very low (even single-digit) counts within large, for example, millilitre sample volumes (for instance, circulating tumour cells in the blood of cancer patients). Suspended cells are typically identified and/or separated based on characteristics such as density, size, dielectric properties or surface markers. A centrifugal device using up to six parallel streams with different densities for cell sorting has been developed by Shiono and co-workers [14-17]. Another density gradient based method to separate white blood cells and measure the cell concentration has been presented by Schaff and colleagues [18]. Morijiri et al. presented a centrifugal implementation of a pinched-flow structure to separate a mixture of beads based on parameters such as size and density [19]. Dielectrophoretic (DEP) cell sorting which relies on differences in dielectric properties has also been successfully applied to cell separation on disc, using an array of carbon [20 $0^{\circ}$ or metal electrodes [21]. All the above described methods rely on differences in the intrinsic physical properties between different cell types. The advantage is that this allows for a label-free separation, eliminating the need for antibodies and complex sample preparation. However, cells often have very similar physical properties and can only be distinguished by characteristic morphologies or surface markers. Methods such as MACS use marker specific antibodies immobilized on paramagnetic beads that bind to the target cells and can subsequently be separated from the background cells by applying a magnetic field. Pamme and co-workers pioneered pressure-driven microfluidic systems using magnetic beads for retrieving target cells from background cells $\left[22,23^{\circ}\right]$ or perform bead-based assays [24].

Recently Kirby $e t$ al. developed a centrifugo-magnetophoretic sorting scheme on a centrifugal microfluidic platform to separate magnetically tagged particles [25 $5^{\bullet \bullet}$ or cells [26] from a background population. Target cells are specifically labelled with magnetic beads. The cell suspension then sediments under stagnant flow conditions and magnetically tagged cells are deflected towards an on-disc magnet and thus separated from the background cells. Chen and colleagues developed a system based on negative selection to separate MCF7 cancer cells from a background of Jurkat cells [27]. In this approach, magnetic microparticles have been coated with anti-bodies specific to Jurkat cells. These magnetically labelled Jurkat cells have then been removed from the suspension in a multi-stage magnet setup. The authors reported a depletion rate of $99.96 \%$ for Jurkat cells and a recovery rate of $60 \pm 10 \%$ for MCF7 cells. In another work from the same group, positive selection using magnetic beads was applied to separate circulating endothelia cells from a background of peripheral blood [28]. Figure 2 shows systems for cell separation using magnetic beads.

\section{Cell capturing and assaying}

Another important step of the process chain is to perform an assay to identify cells and present them for read out. Specifically the capability to trap cells in spatially welldefined locations, expose them to different environmental conditions or reagents and measure the cellular response on a single cell level has attracted much interest. 
Figure 2

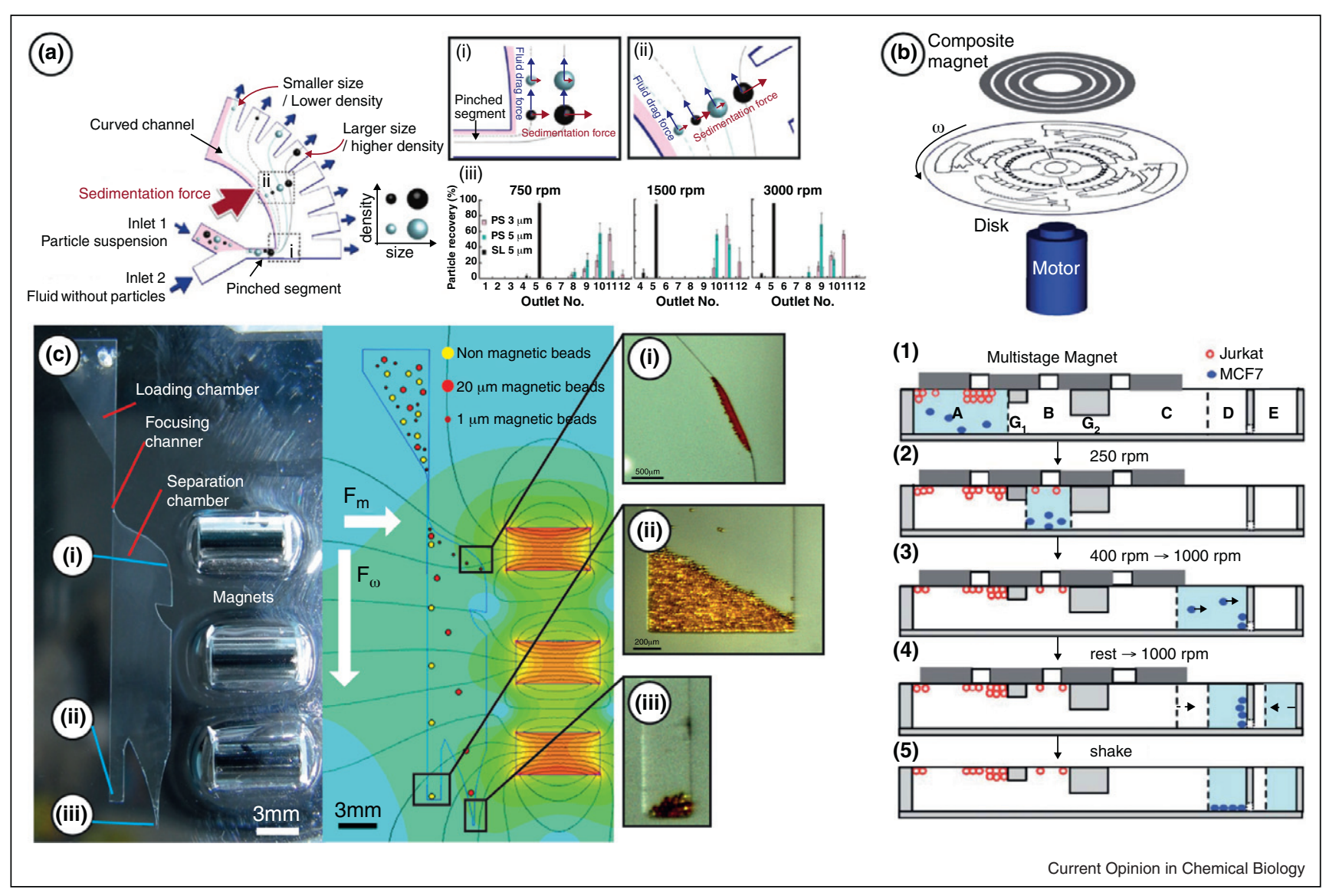

(a) Structure for separating particles according to size and density based on pinched flow. The working principle has been demonstrated using PS and silica beads in different sizes. Large particles with high density are extracted via the first outlets, while smaller particle with lower densities leave at outlets distant from the inlet. Insets I and II show the forces acting on the particles at two different stages of the separation process. The separation results of PS and silica (SL) particles for different rotation frequencies are shown in III. With kind permission from Springer Science+Business Media [19]. (b) Negative separation of MCF7 breast cancer cells from a background of Jurkat cells. The design of the disk and magnet setup is shown on the top while sequence (1)-(5) show the separation process. Jurkat and MCF7 cells were incubated off-disc with magnetic beads labelled with anti-CD45 IgG to specifically bind the beads to the Jurkat cells. The suspension was then introduced in reservoir A (1). Spinning the disc transferred the cell suspension to the outer reservoir $D$ via reservoirs $B$ and $C$, leading to a purification of the cell suspension due to the retention of the magnetically labelled Jurkat cells in the intermediate reservoirs (2)-(4). After separation, shaking the disc spreads the MCF7 cells on the bottom of the chamber to perform counting (5). Reproduced with permission of The Royal Society of Chemistry [27]. (c) Centrifugo-magnetophoretic separation of non-magnetic and magnetic micro beads. The separation chamber is completely filled with PBS buffer before introducing the bead suspension in the loading chamber. Spinning the disc leads to sedimentation of the particles into the separation chamber under stagnant flow conditions, where the beads are exposed to the (essentially) transversal magnetic field generated by the on-disc magnets. During sedimentation magnetic beads are separated by size (insets I and III) and non-magnetic particles sediment on a straight radial pathway into chamber II. With kind permission from Springer Science+Business Media [25*0].

Di Carlo and colleagues presented a pressure-driven system using an array of u-shaped cups to capture different cell types and perform experiments at single-cell level [29,30]. A similar, flow-based system using an array of mechanical traps for cell pairing was presented by Skelley and colleagues [31]. On a centrifugal platform, Kubo and co-workers performed cell trapping in microchambers and demonstrated the implementation of an on-disc cell viability assay [32]. Another implementation of single-cell traps on a centrifugal platform was reported by Lee $e t$ al. [33]. Traps have been aligned along the radially outwards wall of inclined channels. A cell suspension was then flowed through and cells were captured in the traps. Subsequently cytotoxicity studies have been performed on the captured cells. Single cell traps have also been utilized to perform on-disc polymerase chain reaction (PCR) for the detection of Salmonella enterica [34]. Chen et al. reported on a system comprising of a spiral channels with integrated trapping sites for cells. Following capturing, the cells were immobilized in agarose gel and peeled off, thus generating a cell array for off-disc studies [35]. Burger $e t$ al. developed a system using an array of scale matched V-cups to capture microbeads [36 $\left.{ }^{\bullet \bullet}\right]$ and cells [37]. Because of the purely sedimentation based trapping 
method (i.e. in the absence of flow lines) a very high capture efficiency close to $100 \%$ was reported. This platform has been used to perform bead-based immunoassays [ $\left[36^{\circ}\right.$ ] as well as discrimination of captured cells [37]. Very recently, Hattori and Yasuda demonstrated a system based on double Y-shaped channels to transfer single cells between two adjacent liquid streams, thus effectively changing the surrounding medium and consequently exposing the cells to different conditions [38]. A system to measure the cell concentration of a suspension using the optical system of a conventional CD drive has been presented by Imaad and colleagues [39]. The cell suspension is introduced in microchannels on a conventional data CD. The data are then read out using a CD-ROM drive and the error rate generated due to light being scattered on the suspended cells is measured. The authors reported a linear correlation between measured error rate and concentration of cells in the channels. Systems to perform cell assays are shown in Figure 3.

Figure 3

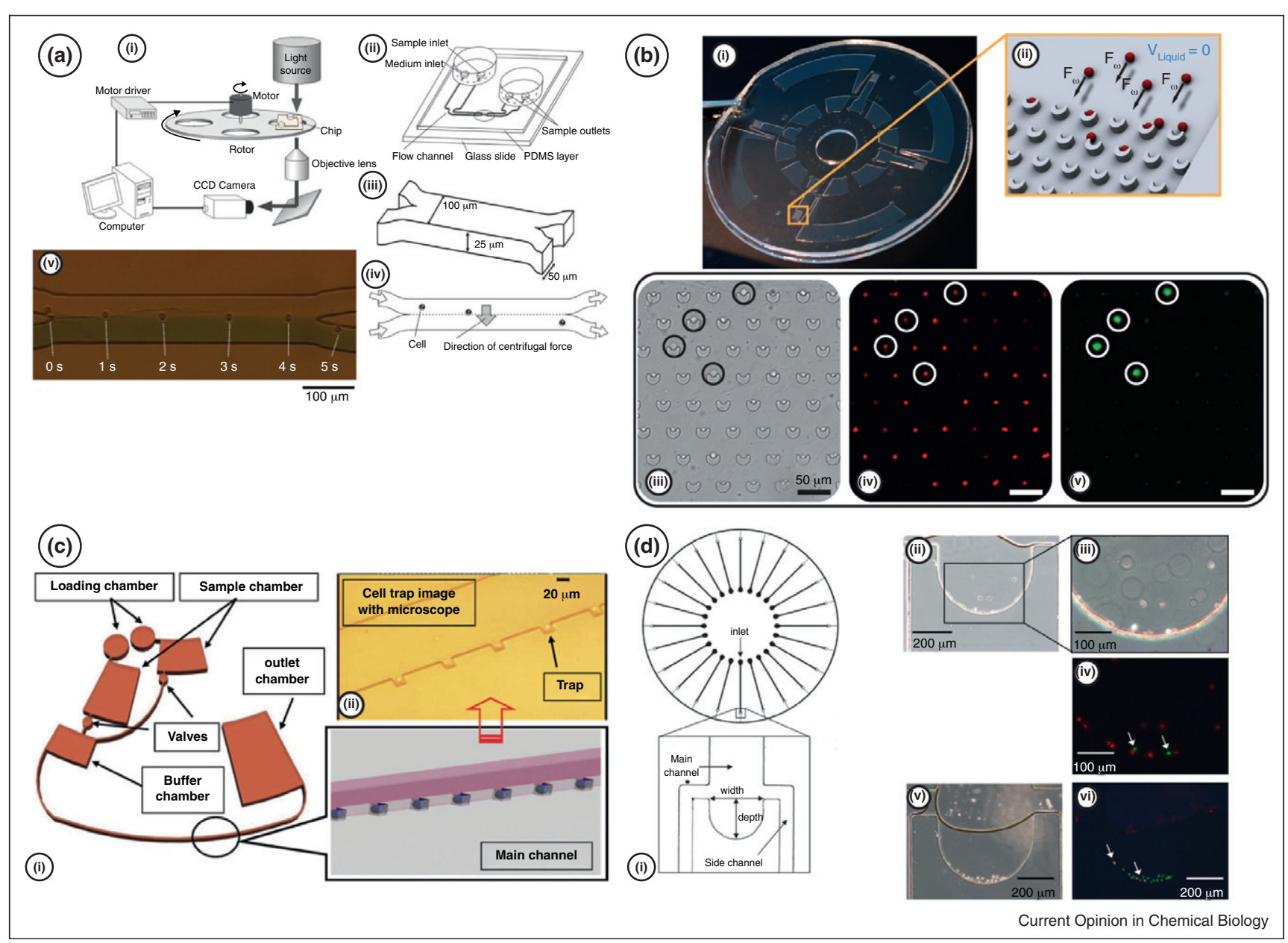

Cell capturing and assaying structures. (a) A chip comprising of reservoirs connected by a double-Y shaped channel. The set-up is shown in (I). (II) Cell suspension is introduced in the radially inwards channel (sample inlet), while a second (cell free) medium is flowing through the radially outwards channel (medium inlet). During rotation, cells sediment from the sample stream into the medium stream, thus allowing rapid exchange of the medium surrounding the cells (IV) and (V). A separation efficiency of $93.5 \%$ has been reported. Reproduced with permission from [38]. (b) V-cup based cell capture scheme. A disc containing four identical cell capturing structures is shown in (I). Cells sediment into the capturing array under stagnant flow conditions and get mechanically trapped in the scale matched V-cup structures (II). Following capturing, the medium in the chamber can be exchanged several times to perform immuno staining of cells. Images (III)-(V) show bright field and fluorescent images of the same array area. A mix of RPMI 8226 and MCF7 has been captured in the array (III). DNA in all cells was then stained with propidium iodide (IV) and MCF7 cells have been identified with FITC labelled anti-EpCAM IgG (V). Reproduced with permission from [37]. (c) Cell capturing structure comprising of an inclined channel with capturing pockets along the radially outwards side wall (I). After flowing a cell suspension through the channel, cells are trapped in the pockets (II). This platform has been utilized to perform cell cytotoxicity experiments. Reprinted with permission from Elsevier [33]. (d) Kubo et al. presented a structure for the capturing of cells (I). Jurkat cells have been captured and subsequently been stained to differentiate living (green) and dead (red) cells (II)-(VI). Reprinted with permission from Elsevier [32]. 


\section{Conclusion and outlook}

The research on centrifugal microfluidic platforms for cell and particle handling is still in its infancy compared to commercially, fully established, technologies such as flow cytometers and multi-well plates. Nevertheless, this 'labon-a-disc' platform bears a high potential to provide advanced tools for cell research as well as for diagnostic point-of-care applications. Centrifugal platforms are especially well suited for applications involving cell handling due to the fact that differences in densities can easily be harnessed for separation purposes (centrifugation based cell removal or separation using standard lab centrifuges are well established), the very simple actuation principle and the clean, modular separation between (disposable) disc and drive/readout unit. Despite these advantages, the centrifugal platform also faces unique challenges, most notably the unidirectional flow due to the centrifugal force which is always pointing away from the centre of rotation, and the difficulty to interface the rotating disc with the stationary instrument (e.g. for power transfer or signal readout). However, we believe that the advantages by far outweigh the drawbacks and, considering the currently on-going research efforts, we believe that the centrifugal platform has the potential to significantly advance point-of-care diagnostics.

\section{Acknowledgements}

This work has been supported by the Science Foundation Ireland under Grant No. 10/CE/B1821 and the Irish Cancer Society Research Fellowship Award CRF10K.

\section{References and recommended reading}

Papers of particular interest, published within the period of review, have been highlighted as:

- of special interest

•• of outstanding interest

1. Andersson $\mathrm{H}$, van den Berg $\mathrm{A}$ : Microfluidic devices for cellomics: a review. Sens Actuators B: Chem 2003, 92:315-325

2. Erickson D, Li D: Integrated microfluidic devices. Anal Chim Acta 2004, 507:11-26.

3. Huh D, Gu W, Kamotani Y, Grotberg J, Takayama S: Microfluidics for flow cytometric analysis of cells and particles. Physiol Meas 2005, 26:R73-R98.

4. Lindstrom $\mathrm{S}$, Andersson-Svahn $\mathrm{H}$ : Overview of single-cell analyses: microdevices and applications. Lab Chip 2010, 10:3363-3372.

5. Lee WC, Bhagat AAS, Huang S, Van Vliet KJ, Han J, Lim CT: Highthroughput cell cycle synchronization using inertial forces in spiral microchannels. Lab Chip 2011, 11:1359-1367.

6. Bhagat AAS, Kuntaegowdanahalli SS, Papautsky I: Continuous particle separation in spiral microchannels using dean flows and differential migration. Lab Chip 2008, 8:1906-1914.

7. Ducrée J, Haeberle S, Lutz S, Pausch S, von Stetten F, Zengerle R: The centrifugal microfluidic bio-disk platform. $J$ Micromech Microeng 2007, 17:S103-S115.

8. Gorkin R, Park J, Siegrist J, Amasia M, Lee BS, Park J, Kim J,

- Kim H, Madou M, Cho Y: Centrifugal microfluidics for biomedical applications. Lab Chip 2010, 10:1758-1773.

A recent review of biomedical centrifugal systems, covering a wide range of the research currently performed.
9. Madou M, Zoval J, Jia G, Kido H, Kim J, Kim N: Lab on a CD. Annu -. Rev Biomed Eng 2006, 8:601-628.

This work provides a comprehensive overview of centrifugal microfluidics, the governing forces and the fundamental unit operations.

10. Burger R, Ducrée J: Handling and analysis of cells and bioparticles on centrifugal microfluidic platforms. Expert Rev Mol Diagn 2012, 12:407-421.

11. Zhang JL, Guo QQ, Liu M, Yang J: A lab-on-CD prototype for high-speed blood separation. J Micromech Microeng 2008, 18 125025 (6 pp.).

12. Häberle S, Brenner T, Zengerle R, Ducrée J: Centrifugal extraction of plasma from whole blood on a rotating disk. Lab Chip 2006, 6:776-781.

13. Li T, Zhang L, Leung KM, Yang J: Out-of-plane microvalves for whole blood separation on lab-on-a-CD. J Micromech Microeng 2010, 20:105024.

14. Shiono H, Ito $\mathrm{Y}$ : Novel method for continuous cell separation by density gradient centrifugation: evaluation of a miniature separation column. Prep Biochem Biotechnol 2003, 33:87-100.

15. Shiono $\mathrm{H}, \mathrm{Okada} \mathrm{T}$, Ito $\mathrm{Y}$ : Application of a novel continuous-flow cell separation method for separation of cultured human mast cells. J Liq Chromatogr Relat Technol 2005, 28:2071-2083.

16. Shiono $\mathrm{H}$, Chen HM, Okada $\mathrm{T}$, Ito $\mathrm{Y}$ : Colony-forming cell assay for human hematopoietic progenitor cells harvested by a novel continuous-flow cell separation method. J Chromatogr $A$ 2007, 1151:153-157.

17. Shiono $\mathrm{H}$, Ogawa $\mathrm{S}$, Matsui $\mathrm{T}$, Niwata $\mathrm{S}$, Okada $\mathrm{T}$, Ito $\mathrm{Y}$ : Preparation of basophils from human peripheral blood by a novel continuous flow-through cell separation method. Physiol Sci 2010, 60:S173.

18. Schaff UY, Tentori AM, Sommer GJ: Differential white cell count by centrifugal microfluidics. 14th International Conference on Miniaturized Systems for Chemistry and Life Sciences ( $\mu$ TAS). 2010:103-105.

19. Morijiri T, Sunahiro S, Senaha M, Yamada M, Seki M: Sedimentation pinched-flow fractionation for size- and density-based particle sorting in microchannels. Microfluid Nanofluid 2011, 11:105-110.

20. Martinez-Duarte R, Gorkin RA, Abi-Samra K, Madou MJ: The - integration of 3D carbon-electrode dielectrophoresis on a CD-like centrifugal microfluidic platform. Lab Chip 2010, 10:1030-1043.

This work shows an interesting approach for cell sorting by combining electrostatic and centrifugal force.

21. Boettcher M, Jaeger MS, Riegger L, Ducrée J, Zengerle R, Duschl C: Lab-on-chip-based cell separation by combining dielectrophoresis and centrifugation. Biophys Rev Lett 2006, 1:443-451.

22. Pamme N, Wilhelm C: Continuous sorting of magnetic cells via on-chip free-flow magnetophoresis. Lab Chip 2006, 6:974-980.

23. Pamme N: Magnetism and microfluidics. Lab Chip 2006, - 6:24-38.

This paper surveys microfluidic systems incorporating magnetic forces for particle handling.

24. Bronzeau S, Pamme N: Simultaneous bioassays in a microfluidic channel on plugs of different magnetic particles. Anal Chim Acta 2008, 609:105-112.

25. Kirby D, Siegrist J, Zavattoni L, Burger R, Ducrée J: Centrifugo-

- magnetophoretic particle separation. Microfluid Nanofluid 2012, http://dx.doi.org/10.1007/s10404-012-1007-6, in press

This work reports on the centrifugo-magnetophoretic particle separation.

26. Siegrist J, Burger R, Kirby D, Zavattoni L, Kijanka G, Ducrée J: Stress-free centrifugo-magnetic 2D-separation of cancer cells in a stopped-flow mode. 15th International Conference on Miniaturized Systems for Chemistry and Life Sciences ( $\mu$ TAS). 2011:1915-1917.

27. Chen C, Chen K, Pan Y, Lee T, Hsiung L, Lin C, Chen C, Lin C, Chiang B, Wo AM: Separation and detection of rare cells in a microfluidic disk via negative selection. Lab Chip 2011, 11:474-483. 
28. Chen K, Lee T, Pan Y, Chiang C, Chen C, Yang Y, Chiang B, Lee H, Wo AM: Detection of circulating endothelial cells via a microfluidic disk. Clin Chem 2011, 57:586-592.

29. Di Carlo D, Lee LP: Dynamic single-cell analysis for quantitative biology. Anal Chem 2006, 78:7918-7925.

30. Di Carlo D, Wu LY, Lee LP: Dynamic single cell culture array. Lab Chip 2006, 6:1445-1449.

31. Skelley AM, Kirak O, Suh H, Jaenisch R, Voldman J: Microfluidic control of cell pairing and fusion. Nat Methods 2009, 6:147-152.

32. Kubo I, Furutani S, Matoba K: Use of a novel microfluidic disk in the analysis of single-cell viability and the application to Jurkat cells. J Biosci Bioeng 2011, 112:98-101.

33. Lee S, Kang JY, Lee I, Ryu S, Kwak S, Shin K, Kim C, Jung H, Kim T: Single-cell assay on CD-like lab chip using centrifugal massive single-cell trap. Sens Actuators A: Phys 2008, 143:64-69.

34. Furutani S, Nagai H, Takamura Y, Kubo I: Compact disk (CD)shaped device for single cell isolation and PCR of a specific gene in the isolated cell. Anal Bioanal Chem 2010, 398:2997-3004.
35. Chen $\mathrm{H}$, Li X, Wang $\mathrm{L}$, Li PCH: A rotating microfluidic array chip for staining assays. Talanta 2010, 81:1203-1208.

36. Burger R, Reith P, Kijanka G, Akujobi V, Abgrall P, Ducrée J: Array-

-. based capture, distribution, counting and multiplexed assaying of beads on a centrifugal microfluidic platform. Lab Chip 2012, 12:1295.

This paper introduces a geometrical trapping scheme for arrayed capture of individual particles on a centrifugal platform and its application for bead-based immunoassays.

37. Burger R, Kijanka G, Sheils O, O’Leary J, Ducrée J: Arrayed capture, assaying and binary counting of cells in a stoppedflow sedimentation mode. 15th International Conference on Miniaturized Systems for Chemistry and Life Sciences ( $\mu$ TAS). 2011:538-540.

38. Hattori A, Yasuda K: Evaluation of a centrifuged double Yshape microfluidic platform for simple continuous cell environment exchange. Int J Mol Sci 2012, 13:819-827.

39. Imaad SM, Lord N, Kulsharova G, Liu GL: Microparticle and cell counting with digital microfluidic compact disc using standard CD drive. Lab Chip 2011, 11:1448-1456. 